\title{
Editorial
}

\section{Antibiotics in Dentistry-When, What and Why?}

Even 75 years after the path-breaking discovery of penicillin by Sir Alexander Fleming, infections continue to be a bane to Human health. This is because infections have evolved with therapy. Overuse, misuse, and abuse of antibiotics have led to increased virulence, antibiotic resistance, and the emergence of new genetic mutants all of which have enabled microbes to outsmart mankind and be a rising threat. The global problem of antibiotic resistance has assumed such dimensions that even the World Health Organization (WHO) considers it a pandemic community issue. It, therefore, becomes the responsibility of every health practitioner be it medical or dental to be judicious in the use of antibiotics and undo the self-inflicted damage.

Because infections which arise as a consequence of caries and periodontal disease are the most common afflictions in dentistry, antibiotic prescriptions have traditionally become an integral part of dental treatment. Little credence has been given to the point that antibiotics are an armor to be used in times of absolute need rather than as a norm. Indiscriminate use has increased the financial burden of treatment, susceptibility to adverse effects and singularly contributed to the risk of development of resistant strains of microbes. This may be of particular relevance to the complex biological ecosystem of the oral cavity with a very fine balance between the pathogenic and nonpathogenic strains living in a biofilm.

A dental clinician needs to respect the old surgical doctrine: "Pus cannot be cured by penicillin". Treatment philosophies should focus on the location and removal of the cause rather than resorting to antibiotic therapy as the sole weapon in this battle with odontogenic infections and the inflammatory processes that spinoff.

Improving our antibiotic use is critical for the safety of the patients and the future of Medicine and Dentistry. In a concerted effort toward the same, antibiotic stewardship programs have been launched worldwide in all specialties. They refer to a set of coordinated strategies to improve the use of antimicrobial medications with the goal of enhancing patient health outcomes, reducing resistance and lowering costs.

The questions that need to be answered with clarity by every dental professional in the process of decision making on antibiotic therapy include:

- What are the clinical conditions that would genuinely benefit from the cover?

- What is the justification for the prescription?

- Which is the most appropriate antibiotic of choice?

- What would be the most effective frequency and length of therapy?

The clinical harbingers which indicate that antibiotic support is necessary for management include:

- Signs of systemic response to infection like fever, malaise, raised respiratory rate and lymphadenopathy

- Breach of local anatomical boundaries and spread into tissue spaces

- Compromised immunity state due to uncontrolled diabetes, long-term corticosteroid therapy, cancers and conditions like cyclic neutropenia and leukemia

- As a prophylactic measure in patients with rheumatic heart disease, solid organ transplants, implants and joint prosthesis which can harbor bacterial vegetations.

This reiterates the fact that majority of the inflammatory presentations in day-to-day dental practice like pain, edema, localized abscesses, and chronic sinus tracts need only elimination of the cause with good debridement and use of antibiotics are superfluous. Procedures for management of uncomplicated endodontic or periodontal lesions, localized walled off abscesses, uncomplicated extraction and placement of single implants in normal healthy individuals with sound defense, clearly stand no reason for antibiotic prophylaxis or therapy.

The second step after narrowing down on the patient population who warrant an antibiotic cover is the selection of the appropriate antibiotics and its regime with dosage and frequency. Once decided, "Waiting is Wasting". Empirical antibiotics from the penicillin group, typically comprising amoxicillin or amoxicillin in combination with clavulanic acid has worked in most odontogenic infections. More serious involvement resolves with the addition of metronidazole and beta-lactamase resistant penicillins. Culture and sensitivity testing may add value only in resistant and rapidly spreading tissue-space infections. Samples for testing should preferably be drawn before initiating the empirical therapy. 
The efficacy of antimicrobials typically has been found to be either concentration or time-dependent. Concentration-dependent drugs like metronidazole have shown a linear relationship between the concentration and cidal activity, implying that even a single high dose could make a difference. On the other hand, time-dependent drugs have demonstrated efficacy only when they have remained in the bloodstream at therapeutic levels for sufficient length of time, making the frequency of administration a key factor. This justifies the administration of amoxicillin in a dose of $250 \mathrm{mg}$ four times a day, rather than a higher dose of $500 \mathrm{mg}$ thrice in adults.

Lack of evidence-based universal antibiotic policies in dentistry account for the present problem of the variety of prescription trends with no standardization. Recent inclinations have been towards opting for short high dose regimes which lower cost and adverse effects.

Diversity accounts for its complexity. Microbial profile, susceptibility characteristics, innate host resistance, and environmental conditions vary between populations and geographical areas. Hence, a one size fits all philosophy may not work and universal guidelines hard to develop. Genetic mutations and continuous evolution of new strains have added a third dimension.

Lessons learned from past experience and evidence have however helped draw the following conclusion:

- Though time and technology have helped understand the microbial characteristics and phenomena better, the battle is still far from being won.

- Every hospital should develop an antibiogram-based policy for therapy with stratification of patients and clear prescription algorithms.

- Antibiotic policies need to be revisited and edited yearly to cater to the changing needs and profiles.

- There are very few definite indications for antibiotic use in dentistry.

- Most odontogenic infections are susceptible and respond well to the penicillin depot and its derivatives.

- The focus should be on the elimination of the cause rather than resort to medical protection.

- Absolute need of the hour is a change in the mindset with realization and insight into the rationale and absolute indications.

Srivalli Natrajan

Professor and Head

Department of Oral and Maxillofacial Surgery

MGM Dental College and Hospital, Mumbai, Maharashtra, India

(Executive Editor, Journal of Contemporary Dentistry) 